\title{
Genetic studies yield clues to the pathogenesis of Langerhans cell histiocytosis
}

\author{
Li Zhang and Joel Moss
}

Affiliation: Cardiovascular and Pulmonary Branch, National Heart, Lung, and Blood Institute, National Institutes of Health, Bethesda, MD, USA.

Correspondence: Joel Moss, Cardiovascular and Pulmonary Branch, National Heart, Lung, and Blood Institute, Room 6D05, Building 10, National Institutes of Health, Bethesda, MD, 20892-1590, USA.

E-mail: mossjanhlbi.nih.gov

○

@ERSpublications

Identifying new therapeutic targets of interstitial lung disease from genetic studies http://ow.ly/ZTv9U

Interstitial lung diseases (ILDs) comprise a heterogeneous group of rare respiratory disorders characterised by diffuse pulmonary infiltrates and destruction of lung parenchyma [1]. With growing evidence that mutations in genes encoding proteins that participate in critical regulatory pathways have roles in the pathogenesis of some forms of ILD, it appears that some diseases under the ILD umbrella may be neoplastic and associated with the presence of cancer-like cells, not found in healthy individuals [2]. For example, lymphangioleiomyomatosis (LAM) results from the proliferation of LAM cells with mutation of tuberous sclerosis (TSC) 1 and TSC2 genes. LAM can be sporadic or occur in association with TSC, an autosomal dominant disorder [3]. TSC2 mutations were detected in seven (50\%) out of 14 and a TSC1 mutation in one (7.1\%) out of 14 TSC-LAM patients [4]. Sporadic LAM appears to be driven by TSC2 mutation [5]. The proteins encoded by TSC1 and TSC2 are upstream of the mammalian target of rapamycin pathway, which has been targeted in LAM treatment (Multicenter International Lymphangioleiomyomatosis Efficacy and Safety of Sirolimus (MILES) trial) [6].

Pulmonary Langerhans cell histiocytosis (PLCH) is a rare ILD that occurs predominantly in young smokers [7-10]. PLCH is considered to be a neoplastic process, which results from the proliferation of tumour cells exhibiting aberrant expression of CD1a on their surface and BRAF-V600E oncogenetic mutations [11].

In this issue of the European Respiratory Journal, MouraH et al. [12] present the remarkable finding that NRAS mutations occur in PLCH lesions. This is the first documentation of NRAS mutations in patients with Langerhans cell histiocytosis (LCH).

Activating mutations are seen at high frequency in genes encoding proteins of the mitogen-activated protein kinase (MAPK) pathway, such as BRAF-V600E in cancer [13]. Extracellular growth factors bind to cell surface transmembrane tyrosine kinase receptors, resulting in activation of RAS, a member of the GTP-binding superfamily of $20-\mathrm{kDa}$ proteins, which then activates a protein kinase cascade (RAS, RAF, MAPK kinase (MEK), extracellular signal-regulated kinase (ERK)) comprising the MAPK signalling pathway, which regulates cell proliferation [14]. BRAF, a member of the RAF protein family, is a serine-threonine kinase. Activating mutations in the BRAF gene have been associated with cancers,

Received: March 182016 | Accepted: March 212016

Support statement: The study was supported by the Intramural Research Program, National Institutes of Health, National Heart, Lung, and Blood Institute, Bethesda, MD, USA. Funding information for this article has been deposited with FundRef.

Conflict of interest: None declared.

The content of this work is not subject to copyright. Design and branding are @ERS 2016. 
including melanomas, colorectal carcinomas and ovarian neoplasms [15]. The BRAF-V600E mutation results in constitutive activation of the serine-threonine kinase and increased cell proliferation [16]. The discovery of the activating mutation in $B R A F$ is consistent with the conclusion that $\mathrm{LCH}$ is a neoplastic process with inflammatory manifestations [17]. ERK activation appears to be a universal event in LCH, resulting from dysregulation of upstream signalling proteins. The RAF/MEK/ERK pathway is involved in several cellular responses including cell cycle regulation, cell proliferation and differentiation, and cell survival and apoptosis [18]. Because activation of the RAF/MEK/ERK signalling pathway was observed in all cases [11], additional activating mutations in other members of this cascade were expected.

MAP2K1 mutations were reported in 2014 in two independent studies. Brown et al. [19] observed that 18 (45\%) out of $40 \mathrm{LCH}$ patients had BRAF mutations whereas $11(27 \%)$ out of 40 had a MAP2K1 mutation. Results of whole exome sequencing of samples from LCH lesions and normal tissues obtained from 41 patients, presented by CHAKRABORTy et al. [20], revealed that $20(50 \%)$ out of 41 cases had a somatic BRAF mutation, while seven other cases harboured MAP2K1 mutations. The MAP2K1 mutations were associated with phosphorylation of ERK. In addition, somatic mutations of the MAPK pathway genes, ARAF and $E R B B 3$, were detected in two individuals [20]. NELSON et al. [21] later reported detection of MAP2K1 and $M A P 3 K 1$ mutations in LCH lesions. In all of these studies, mutations in BRAF or in MAP2K1 were mutually exclusive, with MAP2K1 involved in a minority (25-35\%) of LCH patients [20].

MOURAH et al. [12] again confirmed activation of the MAPK signalling pathway and found the BRAF-V600E mutation in $13(50 \%)$ out of $26 \mathrm{PLCH}$ biopsies, with a MAP2K1 mutation in three $(20 \%)$ out of 13 BRAF wild-type PLCH lesions. A recent study found that $50-60 \%$ of Erdheim-Chester disease (ECD) patients and LCH patients harboured BRAF mutations in the diseased tissue [22]. Thus, LCH and ECD may derive from a common cellular progenitor [22, 23]. EmILE et al. [24] focused on ECD, providing evidence of important similarities to and differences from LCH. In particular, NRAS mutations were detected in three out of 17 ECD BRAF-V600E wild-type patients [24]. MourAH et al. [12] further explored gene mutations in LCH. They used both standard pyrosequencing and highly sensitive E-ice-COLD PCR to discover mutations that underlie LCH pathogenesis. 11 (40\%) out of 26 flow-sorted CD1a-positive cells isolated from PLCH lesions had an NRAS mutation. Importantly, NRAS mutations were found only in CD1a-positive cells, demonstrating the genetic lesion in PLCH. In contrast to other forms of $\mathrm{LCH}$, smoking may be the stimulus for PLCH $[8,10]$. Phosphatidylinositol 3-kinase (PI3K)/AKT is one of the main downstream effectors of the RAS family, regulating metabolism, growth, proliferation, survival, transcription and protein synthesis [14,25]. Activating mutations of NRAS have been associated with lung cancer [26], melanoma [27], colorectal cancer [28] and acute myeloid leukaemia [29]. MourAH et al. [12] also showed activation of the AKT pathway in all PLCH cases, a functional consequence of the NRAS mutation. This finding raises the possibility of combined therapy in LCH targeting the RAS/RAF/PI3K/ AKT and BRAF/MEK/ERK pathways.

HUTTER et al. [30] reported that the NOTCH signalling pathway was involved in an LCH lesion. Activated NOTCH1 was detected by Western blot in protein lysates from $10(80 \%)$ out of $12 \mathrm{LCH}$ biopsies (bone, skin and mucosa). Molecular analysis revealed that both isolated and in-tissue LCH cells selectively expressed the NOTCH ligand Jagged 2 (JAG2). They further showed that JAG2 signalling induced key $\mathrm{LCH}$-cell markers in monocyte-derived dendritic cells, suggesting a role of NOTCH signalling in LCH oncogenesis. Therefore, in selected patients, interference with NOTCH signalling might be a potential strategy for LCH treatment [30].

MouraH et al. [12] also identified concurrent BRAF-V600E and NRAS mutations in seven (27\%) out of 26 $\mathrm{PLCH}$ lesions, concluding that they were derived from different clonal populations. Using univariate analyses, they found that clinical outcomes were better for patients with only single BRAF or NRAS mutations than for those with both. Although more studies are needed, a trend for worse pulmonary function is observed for patients with BRAF-V600E mutations than those with NRAS mutations and BRAF wild type.

In summary, the identification of new therapeutic targets may lead to successful treatments for more members of this family of lung diseases. The effectiveness of sirolimus in stabilising lung function, reducing the sizes of angiomyolipomas, and chylous effusions, as well as clearing circulating LAM cells is proof of concept that therapy targeting defective genetic and biochemical pathways can be successful $[3,6$, 31-33]. Targeted therapy is currently used in the management of patients with advanced nonsmall cell lung cancer (NSCLC). Mutations in the epidermal growth factor receptor (EGFR) tyrosine kinase are observed in NSCLC adenocarcinomas; the presence of an EGFR mutation confers a more favourable prognosis and strongly predicts sensitivity to EGFR tyrosine kinase inhibitors [34]. Personalised medical therapies may similarly be found for other lung diseases. Here, MouraH et al. [12] highlight NRAS as an important target in the search for novel molecular therapies. Discovering additional different mutations beyond BRAF-V600E is no doubt a worthwhile means to improve patient care. 


\section{References}

$1 \quad$ Cottin V. Interstitial lung disease. Eur Respir Rev 2013; 22: 26-32.

2 Archontogeorgis K, Steiropoulos P, Tzouvelekis A, et al. Lung cancer and interstitial lung diseases: a systematic review. Pulm Med 2012; 2012: 315918.

3 Harari S, Torre O, Cassandro R, et al. The changing face of a rare disease: lymphangioleiomyomatosis. Eur Respir J 2015; 46: 1471-1485

4 Strizheva GD, Carsillo T, Kruger WD, et al. The spectrum of mutations in TSC1 and TSC2 in women with tuberous sclerosis and lymphangiomyomatosis. Am J Respir Crit Care Med 2001; 163: 253-258.

5 Cai X, Pacheco-Rodriguez G, Fan QY, et al. Phenotypic characterization of disseminated cells with TSC2 loss of heterozygosity in patients with lymphangioleiomyomatosis. Am J Respir Crit Care Med 2010; 182: 1410-1418.

6 McCormack FX, Inoue Y, Moss J, et al. Efficacy and safety of sirolimus in lymphangioleiomyomatosis. $N$ Engl $J$ Med 2011; 364: 1595-1606.

7 Vassallo R, Ryu JH, Colby TV, et al. Pulmonary Langerhans'-cell histiocytosis. N Engl J Med 2000; 342: 1969-1978.

8 Ryu JH, Colby TV, Hartman TE, et al. Smoking-related interstitial lung diseases: a concise review. Eur Respir J $2001 ; 17: 122-132$

9 Sundar KM, Gosselin MV, Chung HL, et al. Pulmonary Langerhans cell histiocytosis: emerging concepts in pathobiology, radiology, and clinical evolution of disease. Chest 2003; 123: 1673-1683.

10 Tazi A. Adult pulmonary Langerhans' cell histiocytosis. Eur Respir J 2006; 27: 1272-1285.

11 Badalian-Very G, Vergilio JA, Degar BA, et al. Recurrent BRAF mutations in Langerhans cell histiocytosis. Blood 2010; 116: 1919-1923.

12 Mourah S, How-Kit A, Meignin V, et al. Recurrent NRAS mutations in pulmonary Langerhans cell histiocytosis. Eur Respir J 2016; 47: 1785-1796.

13 Garnett MJ, Marais R. Guilty as charged: B-RAF is a human oncogene. Cancer Cell 2004; 6: 313-319.

14 Rajalingam K, Schreck R, Rapp UR, et al. Ras oncogenes and their downstream targets. Biochim Biophys Acta 2007; 1773: 1177-1195.

15 Davies H, Bignell GR, Cox C, et al. Mutations of the BRAF gene in human cancer. Nature 2002; 417: 949-954.

16 Cantwell-Dorris ER, O'Leary JJ, Sheils OM. BRAFV600E: implications for carcinogenesis and molecular therapy. Mol Cancer Ther 2011; 10: 385-394.

17 Rizzo FM, Cives M, Simone V, et al. New insights into the molecular pathogenesis of Langerhans cell histiocytosis. Oncologist 2014; 19: 151-163.

18 McCubrey JA, Steelman LS, Chappell WH, et al. Roles of the Raf/MEK/ERK pathway in cell growth, malignant transformation and drug resistance. Biochim Biophys Acta 2007; 1773: 1263-1284.

19 Brown NA, Furtado LV, Betz BL, et al. High prevalence of somatic MAP2K1 mutations in BRAF V600E-negative Langerhans cell histiocytosis. Blood 2014; 124: 1655-1658.

20 Chakraborty R, Hampton OA, Shen X, et al. Mutually exclusive recurrent somatic mutations in MAP2K1 and BRAF support a central role for ERK activation in LCH pathogenesis. Blood 2014; 124: 3007-3015.

21 Nelson DS, van Halteren A, Quispel WT, et al. MAP2K1 and MAP3K1 mutations in Langerhans cell histiocytosis. Genes Chromosomes Cancer 2015; 54: 361-368.

22 Haroche J, Charlotte F, Arnaud L, et al. High prevalence of BRAF V600E mutations in Erdheim-Chester disease but not in other non-Langerhans cell histiocytoses. Blood 2012; 120: 2700-2703.

23 Badalian-Very G. A common progenitor cell in LCH and ECD. Blood 2014; 124: 991-992.

24 Emile JF, Diamond EL, Hélias-Rodzewicz Z, et al. Recurrent RAS and PIK3CA mutations in Erdheim-Chester disease. Blood 2014; 124: 3016-3019.

25 Castellano E, Downward J. RAS interaction with PI3K: more than just another effector pathway. Genes Cancer 2011; 2: 261-274.

26 Ohashi K, Sequist LV, Arcila ME, et al. Characteristics of lung cancers harboring NRAS mutations. Clin Cancer Res 2013; 19: 2584-2591.

27 Kelleher FC, McArthur GA. Targeting NRAS in melanoma. Cancer J 2012; 18: 132-136.

28 Irahara N, Baba Y, Nosho K, et al. NRAS mutations are rare in colorectal cancer. Diagn Mol Pathol 2010; 19: $157-163$.

29 Bacher U, Haferlach T, Schoch C, et al. Implications of NRAS mutations in AML: a study of 2502 patients. Blood 2006; 107: 3847-3853.

30 Hutter C, Kauer M, Simonitsch-Klupp I, et al. Notch is active in Langerhans cell histiocytosis and confers pathognomonic features on dendritic cells. Blood 2012; 120: 5199-5208.

31 Cai X, Pacheco-Rodriguez G, Haughey M, et al. Sirolimus decreases circulating lymphangioleiomyomatosis cells in patients with lymphangioleiomyomatosis. Chest 2014; 145: 108-112.

32 Goldberg HJ, Harari S, Cottin V, et al. Everolimus for the treatment of lymphangioleiomyomatosis: a phase II study. Eur Respir J 2015; 46: 783-794.

33 Taveira-DaSilva AM, Hathaway O, Stylianou M, et al. Changes in lung function and chylous effusions in patients with lymphangioleiomyomatosis treated with sirolimus. Ann Intern Med 2011; 154: 797-805.

34 Sculier JP, Berghmans T, Meert AP. Advances in target therapy in lung cancer. Eur Respir Rev 2015; 24: 23-29. 